\title{
Tophaceous Gout
}

A 79-year-old man presented with right upper quadrant pain at the emergency department. A computed tomography (CT) study of the abdomen and pelvis was performed, which demonstrated multiple calcified gallstones within the gallbladder and features of acute cholecystitis. The patient underwent cholecystectomy and recovered well in the ward. However, during his inpatient stay, he started complaining of severe pain over his left gluteal region.

What does the CT of his pelvis demonstrate? What is the likely diagnosis?
A. Osteosarcoma
B. Myositis ossificans
C. Tumoral calcinosis
D. Metastasis
E. Tophaceous gout

A review of the $\mathrm{CT}$ abdomen and pelvis demonstrated a lobulated, amorphously calcified soft tissue mass located medial and deep to the left gluteus maximus muscle with possible mild erosion seen at the left ischial tuberosity, at the region of the ischiogluteal bursa (Fig. 1). Since the CT study was originally acquired as a dual-energy CT (DECT), reconstruction was performed and monosodium urate crystals were identified within the soft tissue mass (Figs. 2 and 3). The patient underwent serum uric acid and creatinine tests which were in keeping with the diagnosis of gout.

The use of DECT allowed for an accurate diagnosis in this case due to the presence of monosodium urate crystal uptake. Osteosarcoma is a less likely differential as the lesion was centred within the soft tissues. Myositis ossificans would show a zonal pattern of calcifications, which was not seen in this case. The location at the ischiogluteal bursa ${ }^{1}$ would make tumoral calcinosis or calcium hydroxyapatite deposition disease key considerations if not for the presence of DECT. Metastasis is unlikely given the predominant soft tissue calcification with no soft tissue component.

The utilisation of DECT for detection of gout has been well documented. In this case, the availability of DECT enabled the diagnosis to be clinched without any further biopsy or aspiration. The current gold standard for gout

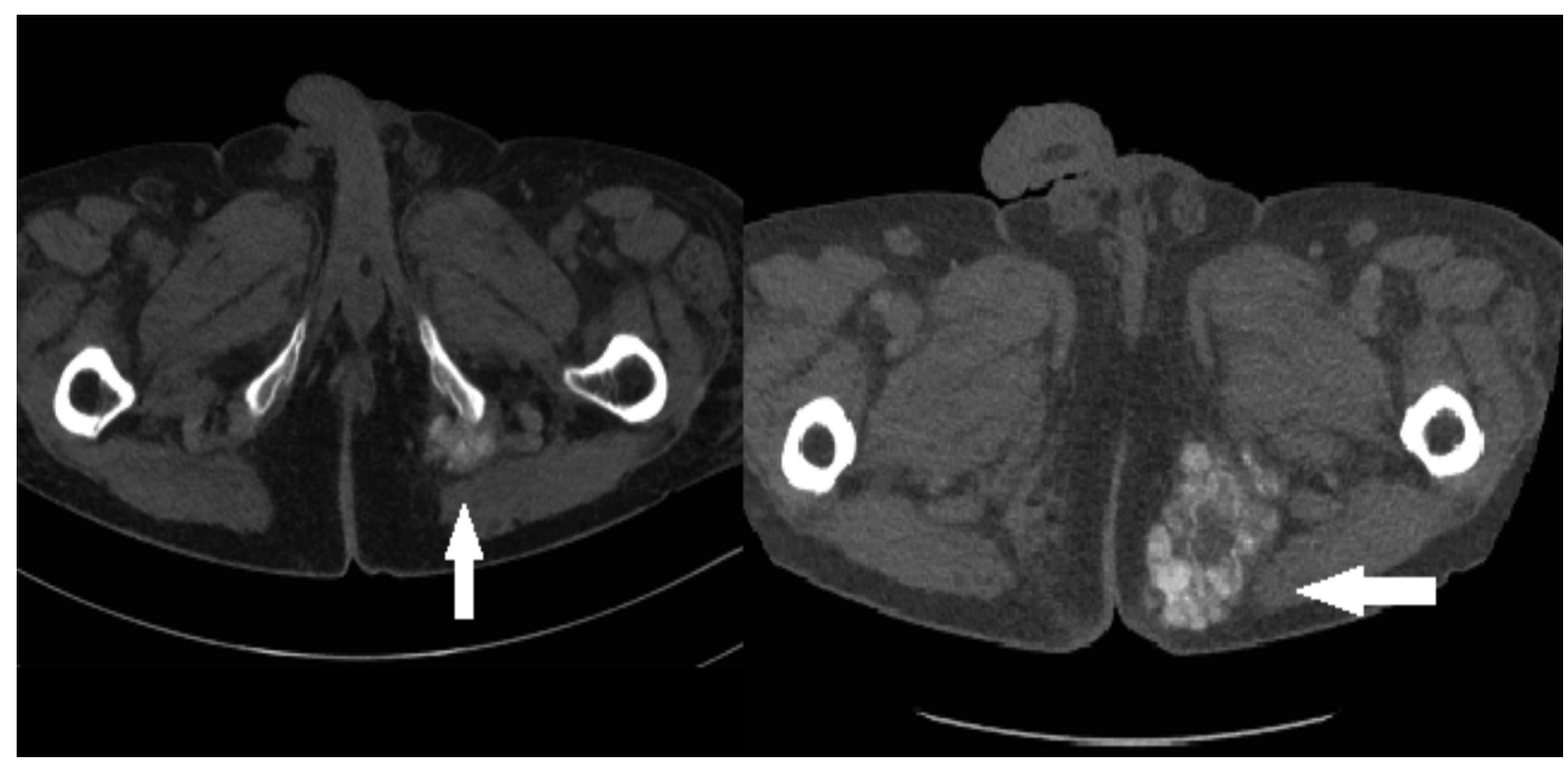

Fig. 1. Axial CT images demonstrate a lobulated soft tissue mass with amorphous calcifications located medial and deep to the left gluteus maximus muscle. The lesion appears to be eroding the left ischial tuberosity. 


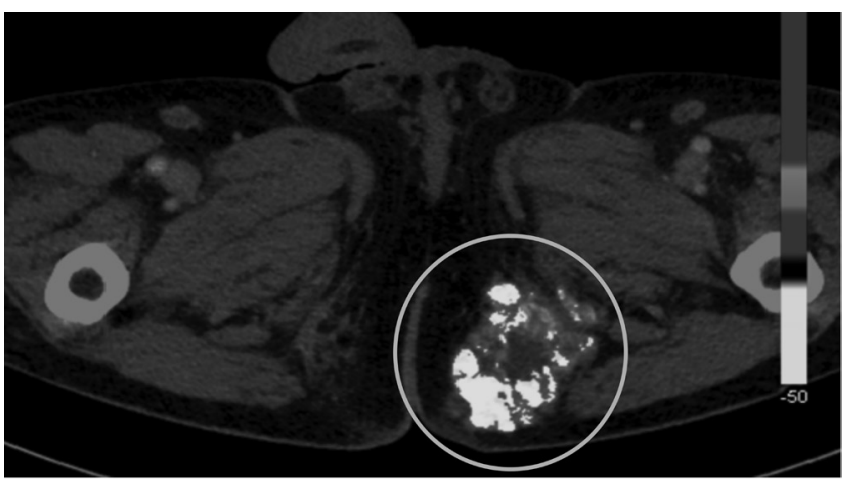

Fig. 2. Dual-energy CT reconstruction with a gout protocol demonstrates extensive monosodium urate crystal deposition within the mass. Gout/ monosodium urate crystals are circled.

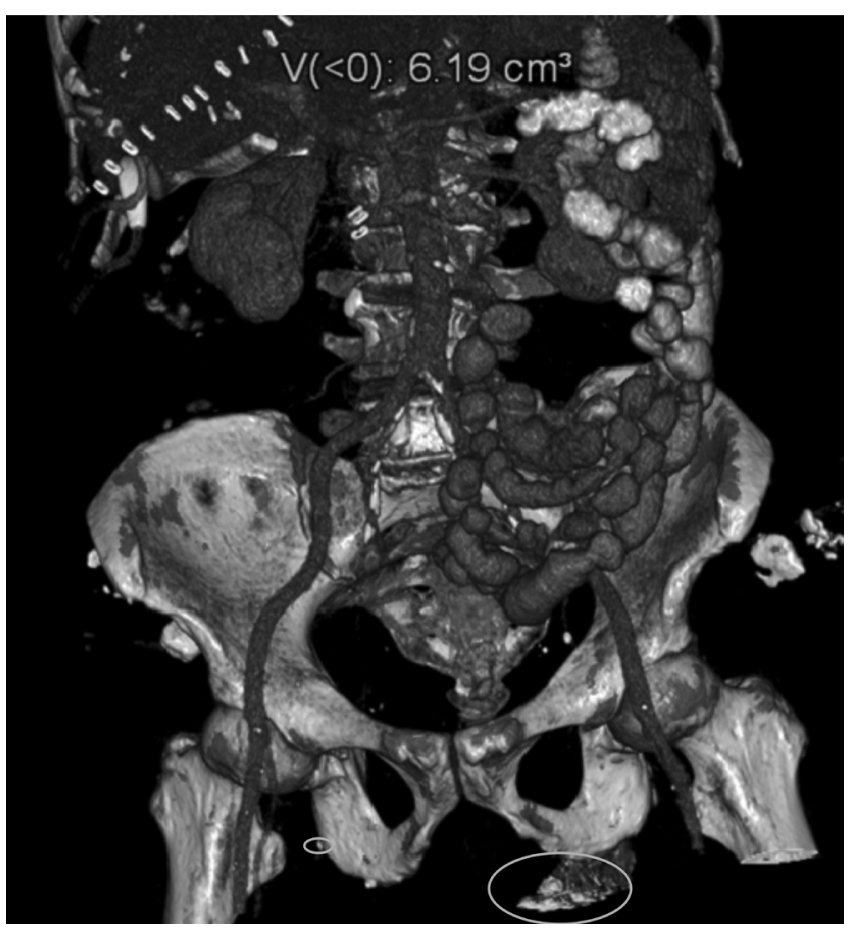

Fig. 3. 3D reconstruction demonstrates the mass with abundant monosodium urate deposition at the region of the left ischial tuberosity. Right upper abdominal surgical clips are seen due to recent cholecystectomy. Some green artifacts (2 circled areas) are seen over the liver, which can occur due to noise, beam hardening or calcifications.

diagnosis is with joint fluid aspiration for monosodium urate crystal analysis via polarisation microscopy. However, joint aspiration is invasive with a risk of complications and may be unreliable when there is only a minimal joint effusion. Furthermore, it is estimated that only $3 \%$ of primary care gout patients actually undergo joint aspiration. ${ }^{2}$ The advent of DECT thus offers the clinician a non-invasive and accurate method of assessing for gout.

Gout is the most common inflammatory arthropathy in men and women. ${ }^{3}$ It typically occurs in those above 40 years of age with a strong male predilection. ${ }^{4}$ There are 5 recognised stages of gout: asymptomatic hyperuricemia, acute gouty arthritis, intercritical gout, chronic tophaceous gout and gouty nephropathy. It usually has an asymmetrical polyarticular distribution and most commonly affects the first metatarsophalangeal joints. Gout can involve the bone, tendon and bursa. Besides musculoskeletal manifestations, long-term sequelae of gout include nephropathy and cardiovascular disease. Thus, prompt and accurate diagnosis is needed for adequate treatment.

Radiographically, the typical appearance is that of welldefined 'punched-out' erosion with sclerotic margins in a marginal and juxta-articular distribution with overhanging edges. Soft tissue amorphous calcifications are another manifestation, while tophaceous gout is pathognomonic. However, these findings are typically seen in late stage gout, and in the early acute setting, plain radiographs have proven to be inadequate for early detection. Ultrasound can have characteristic findings but is operator dependent, and MRI can show bone and soft tissue abnormalities due to gout, although it remains non-specific. DECT has advantages over other modalities due to the ability to quantify urate crystal deposits in the joints/tissues with high sensitivity and specificity. ${ }^{5}$

DECT has been shown to be both sensitive and specific in identifying monosodium urate deposition. ${ }^{2,6}$ The sensitivity rates have been reported to range from $78-100 \%$ and the specificity ranges from $89-100 \%$. DECT, unlike conventional single-energy $\mathrm{CT}$ uses two separate $\mathrm{x}$-ray photon spectra to image simultaneously, allowing interrogation of materials that have different attenuation properties at different energies. Utilising a 2-tissue decomposition algorithm, the differences between calcium and urate's photoelectric effect creates measurable differences in attenuation between urate, calcium and bone at different energies. The different materials are differentiated, colour-coded and superimposed atop a standard grayscale CT, allowing for urate crystal detection and mapping to the anatomical structure. DECT is particularly useful in diagnostic situations where the crystal analysis is non-diagnostic due to inadequate fluid, or if aspiration is difficult or contraindicated.

The utilisation of DECT in the musculoskeletal system is not limited to the use of gout detection. Current usage includes bone marrow edema detection, metal artifact reduction and tendon analysis with potential applications in bone densitometry, arthrography and metastases surveillance. ${ }^{2}$

\section{REFERENCES}

1. Schuh A, Narayan CT, Schuh R, et al. Calcifying Bursitis ischioglutealis: A Case report. J Orthop Case Rep 2011;1:16-8.

2. Mallinson P, Coupal T, McLaughlin P, et al. Dual-Energy CT for the Musculoskeletal System. Radiology 2016;281:690-707. 
3. Mikuls TR, Farrar JT, Bilker WB, et al. Gout epidemiology: results from the UK General Practice Research Database, 1990-1999. Ann Rheum Dis 2005;64:267-72.

4. Koh WH, Seah A, Chai P. Clinical presentation and disease associations of gout: a hospital-based study of 100 patients in Singapore. Ann Acad Med Singap 1998;27:7-10.

5. Girish G, Glazebrook KN, Jacobson JA. Advanced Imaging in Gout. AJR Am J Roentgenol 2013;201:515-25.

6. Bongartz T, Glazebrook KN, Kavros SJ, et al. Dual-energy CT for the diagnosis of gout: an accuracy and diagnostic yield study. Ann Rheum Dis 2015;74:1072-7.
Eugene Low, ${ }^{1} M B B S, F R C R$, Hugue Ouellette, ${ }^{2} M D, F R C P C, A B R$, Peter L Munk, ${ }^{2}$ MDCM, FRCPC, FSIR

${ }^{1}$ Department of Diagnostic Imaging, Tan Tock Seng, Singapore

${ }^{2}$ Diagnostic Radiology Department, Vancouver General Hospital, University of British Columbia, Canada

Address for Correspondence: Dr Eugene Low, Department of Diagnostic Radiology, Tan Tock Seng Hospital, 11 Jalan Tan Tock Seng, Singapore 308133. Email: low_eugene@hotmail.com 